\title{
Critical torsional modes of convection in rotating fluid spheres at high Taylor numbers
}

\author{
J. Sánchez ${ }^{1}$, F. Garcia ${ }^{1}$ and M. Net $^{1} \dagger$ \\ ${ }^{1}$ Applied Physics Department, Universitat Politècnica de Catalunya Jordi Girona Salgado 3. \\ Campus Nord, Mòdul B4, 08034 Barcelona. Spain.
}

(Received $\mathrm{xx}$; revised $\mathrm{xx}$; accepted $\mathrm{xx}$ )

A numerical study of the onset of convection in rotating, internally heated, selfgravitating fluid spheres is presented. The exploration of the stability of the conduction state versus the Taylor and the Prandtl numbers supplies a detailed idea of the laws that fulfil the four types of solutions obtained at low Prandtl numbers. The main result found is that axisymmetric (torsional) modes of convection are preferred at high Taylor numbers in the zero-Prandtl-number limit. This instability appears at low Rayleigh numbers and gives rise to an oscillating single vortex of very high frequency.

\section{Introduction}

The linear theory for the onset of thermal convection in rotating fluid spheres and spherical shells with moderate and large Prandtl numbers, $\sigma$, is well understood from contributions from the late sixties to the present decade, for either stress-free or nonslip boundary conditions (see, for instance, Roberts 1968; Busse 1970; Carrigan \& Busse 1983; Yano 1992; Zhang 1992; Jones et al. 2000; Busse 2002; Dormy et al. 2004; AlShamali et al. 2004; Garcia et al. 2008; Bassom et al. 2011). However, a fewer number of studies were devoted to studying the same problem for very low $\sigma$ and, in particular, the zero-Prandtl-number limit.

When $\sigma$ is small enough, the heating of a fluid excites and maintains inertial oscillations, but simple asymptotic scalings for $T a \rightarrow \infty$ do not exist. In this context, some authors first looked for solutions of the Poincaré equation in rotating spherical geometry. In a paper by Zhang (1993) symmetric and antisymmetric equatorially-attached modes of convection (EA), which travelled azimuthally, in either in the prograde or the retrograde direction, were found. In any of the cases, the latitudinal and radial radii of the waves were $r_{l}=(2 / m)^{1 / 2}$ and $r_{r}=(1 / m)$, respectively, $m$ being the azimuthal wave number. Moreover, Zhang et al. (2001) obtained general analytical expressions for this problem and demonstrated that the internal viscous dissipation of all of the internal waves vanishes for a rotating fluid sphere.

By perturbing the symmetric inertial waves of the Poincaré equation, and considering the Ekman boundary layers, Zhang (1994) obtained EA modes of convection, even for $\sigma=0$. These modes are excited when the thermal energy is able to balance the viscous dissipation. Later, Busse \& Simitev (2004) considered stress-free and thermally insulating boundary conditions, and derived, also by means of a perturbation analysis of the inertial waves, explicit expressions for the dependence of the Rayleigh number on $m$. They found that the azimuthal wave number of the critical Rayleigh number was $m=1$ for very low $\sigma$.

Zhang \& Liao (2004) developed a theory trying to unify the convective instability and

$\dagger$ Email address for correspondence: marta.net@upc.edu 
the inertial-oscillation problems for stress-free boundary conditions. The new asymptotic method covered the asymptotically large $T a$ limit for $0 \leqslant \sigma \sqrt{T a}<\infty$. They considered that in any case the flow is quasi-geostrophic, and wrote the leading-order velocities as either a single quasi-geostrophic inertial-wave mode (EA mode) or a superposition of several of them for the spiralling modes (SP modes). They also took into account the existence of the Ekman boundary layer flow, which controlled the interior flow for low and moderate $\sigma$, or modified it when $\sigma$ is moderately large. More recently the same type of study was extended in Zhang et al. (2007) to non-slip boundary conditions.

This paper revisits the onset of convection for very low Prandtl and high Taylor numbers, taking into account the possibility of having inertial oscillations, i.e. preferred axisymmetric patterns of convection, mainly in the zero-Prandtl-number limit. The remainder of the paper is organised as follows. In $\S 2$ the equations and numerical method employed are briefly introduced. In $\S 3$ the Taylor number dependence of the critical parameters for low Prandtl numbers is studied, and in $\S 4$ and 5 their Prandtl number dependence is investigated for large Taylor numbers. Finally, a summary and a discussion on the main results is included.

\section{The method}

To study numerically the onset of convection in fluid spheres, internally heated and subject to radial gravity $\mathbf{g}=-\gamma \boldsymbol{r}$, with $\gamma>0$, we apply the Boussinesq approximation to the mass, momentum and energy equations, written in the rotating frame of reference of the sphere. We also neglect the centrifugal force since $\Omega^{2} / \gamma \ll 1$ as happens, for instance, in the major planets, and we take constant density in the Coriolis term. In addition, we consider the radius, $r_{o}$, of the sphere as the longitudinal scale, a viscous time scale $r_{o}^{2} / \nu$, and $\nu^{2} / \gamma \alpha r_{o}^{4}$ as the temperature scale. The physical constants are the kinematic viscosity, $\nu$, and the thermal expansion coefficient $\alpha$.

To reduce the number of equations, the velocity field is written in terms of toroidal and poloidal velocity potentials, i.e. $\boldsymbol{v}=\boldsymbol{\nabla} \times(\Psi \boldsymbol{r})+\boldsymbol{\nabla} \times \boldsymbol{\nabla} \times(\Phi \boldsymbol{r})$, and the equations are linearized around the conduction state $\boldsymbol{v}=\mathbf{0}$ and $T_{c}(r)$, with $T_{c}(r)=T_{0}+\mathcal{S}\left(r_{o}^{2}-r^{2}\right) / 6 \kappa$. The constant $S$ is $q / c_{p}, q$ being the rate of internal heat generation per unit mass, $c_{p}$ the specific heat at constant pressure, and $\kappa$ the thermal diffusivity. The equations are

$$
\begin{aligned}
\left(\partial_{t}-\nabla^{2}\right) \mathcal{L}_{2} \Psi & =2 T a^{1 / 2}\left(\partial_{\varphi} \Psi-\mathcal{Q} \Phi\right) \\
\left(\partial_{t}-\nabla^{2}\right) \mathcal{L}_{2} \nabla^{2} \Phi & =2 T a^{1 / 2}\left(\partial_{\varphi} \nabla^{2} \Phi+\mathcal{Q} \Psi\right)-\mathcal{L}_{2} \Theta, \\
\left(\sigma \partial_{t}-\nabla^{2}\right) \Theta & =R a \mathcal{L}_{2} \Phi
\end{aligned}
$$

where $\Theta(r, \theta, \varphi)=T(r, \theta, \varphi)-T_{c}(r)$ is the temperature perturbation and $(r, \theta, \varphi)$ are the spherical coordinates, $\theta$ measuring the colatitude. The operators $\mathcal{L}_{2}$ and $\mathcal{Q}$ are defined as $\mathcal{L}_{2}=-r^{2} \nabla^{2}+\partial_{r}\left(r^{2} \partial_{r}\right)$ and $\mathcal{Q}=r \cos \theta \nabla^{2}-\left(\mathcal{L}_{2}+r \partial r\right)\left(\cos \theta \partial r-r^{-1} \sin \theta \partial \theta\right)$.

The non-dimensional parameters are the internal Rayleigh, Prandtl and Taylor numbers, defined as

$$
R a=\frac{S \gamma \alpha r_{o}^{6}}{3 \kappa^{2} \nu}, \quad \sigma=\frac{\nu}{\kappa} \quad \text { and } \quad T a^{1 / 2}=\frac{\Omega r_{o}^{2}}{\nu}
$$

respectively.

Finally, stress-free boundary conditions $\Phi=\partial_{r r} \Phi=\partial_{r}(\Psi / r)=0$, and a perfectly conducting boundary, which means $\Theta=0$ at $r=r_{o}$, are taken. At $r=0$ regularity conditions are enforced since a collocation method in a Gauss-Lobatto mesh of $N+1$ 


$\begin{array}{lccccc} & T a \times 10^{10} & \sigma & R a_{c} \times 10^{5} & \omega_{c} \times 10^{2} & m_{c} \\ \text { ZL04 } & 0.01 & 0.7 & 21.77 & -3.912 & 7 \\ \text { SGN15 } & 0.01 & 0.7 & 21.76 & -3.909 & 7 \\ \text { ZL04 } & 1 & 0.7 & 424.1 & -19.056 & 16 \\ \text { SGN15 } & 1 & 0.7 & 424.0 & -19.050 & 16 \\ \text { ZL04 } & 1 & 0.05 & 94.00 & -56.46 & 8 \\ \text { SGN15 } & 1 & 0.05 & 91.75 & -56.11 & 8 \\ \text { ZL04 } & 1 & 0.023 & 30.26 & -249.2 & 8 \\ \text { SGN15 } & 1 & 0.023 & 31.92 & -252.5 & 7 \\ \text { ZLB07 } & 0.01 & 0.1 & 9.534 & -7.880 & 5 \\ \text { SGN15 } & 0.01 & 0.1 & 9.641 & -7.855 & 5 \\ \text { ZLB07 } & 0.01 & 0.01 & 3.650 & -18.54 & 2 \\ \text { SGN15 } & 0.01 & 0.01 & 3.699 & -18.51 & 2\end{array}$

TABLE 1. Comparison of the critical parameters $R a_{c}, \omega_{c}$ and $m_{c}$, for stress-free boundary conditions with preceding results of Zhang \& Liao (2004), and for non-slip boundary conditions with Zhang et al. (2007).

points is used to discretize the equations radially. Radial derivative matrices and radial differential operators are built incorporating all of these conditions.

On the sphere the equations for $X=(\Phi, \Psi, \Theta)$ are solved by expanding the functions in spherical harmonic series up to degree $L$, namely

$$
X(r, \theta, \varphi, t)=\sum_{l=0}^{L} \sum_{m=-l}^{l} X_{l}^{m}(r, t) Y_{l}^{m}(\theta, \varphi),
$$

with $Y_{l}^{m}(\theta, \varphi)=P_{l}^{m}(\cos \theta) e^{i m \phi}$, and $P_{l}^{m}$ being the normalised associated Legendre functions of degree $l$ and order $m$. In this way the system (2.1)-(2.3) decouples for each order $m$, and systems, $\dot{X}_{m}=\mathcal{A}_{m} X_{m}$, of $3(L-m+1)(N-1)$ complex ODEs are obtained.

The matrices $\mathcal{A}_{m}$ are block-tridiagonal due to the structure of $\mathcal{Q}$, with $(L-m+1)$ rows of blocks of dimension $3(N-1)$. The onset of convection is given by the instability of the zero solution of these systems. To find the leading spectrum of $A_{m}$ (maximal real part), shift-invert preconditioners are employed. The eigenvalue problem $A_{m} u=\lambda u$ is transformed into $\left(A_{m}-\zeta I\right)^{-1} u=\mu u$, where $\zeta$ is a complex shift. The eigenvectors of both problems are the same, and the eigenvalues are related by $\lambda=\zeta+1 / \mu$. To find the $\mu$ values of largest modulus the ARPACK package, based on Arnoldi algorithms (see Lehoucq \& Sorensen 1996), is used. The linear systems with matrix $\left(A_{m}-\zeta I\right)$ are solved by means of an adapted LU decomposition. If $\zeta=\rho+i \omega$ with $\rho \gtrsim 0$ we obtain the eigenvalues $\lambda=\lambda_{r}+i \lambda_{i}$ closest to $i \omega$. A sequence of imaginary parts $\omega$ is examined, using the information from nearby calculations, to ensure that no modes close to critical are missed. For the same reason, if an azimuthal wave number $m_{0}$ is preferred for some value of the parameters, an interval $\left[m_{0}-\Delta m, m_{0}+\Delta m\right]$ is also explored, and a finer mesh of $\omega$ values is used from time to time. The critical values of the parameters are found by applying a root-finding algorithm to the equation $\lambda_{r}=0$. For any set of parameters the truncation constants $N$ and $L$ have been taken to bound the errors below $1 \%$. The maximal values used for the highest $m$ were $N=80$ and $L=180$. More detailed information on the method used can be found in Sánchez et al. (2016).

The instability of the conduction state is always via a Hopf bifurcation. It gives rise to waves travelling azimuthally when $m_{c}>0$, and oscillations of the fluid if $m_{c}=0$. When 
$m_{c}>0$, the negative frequencies give positive drifting velocities $c=-\omega_{c} / m_{c}$, i.e. the waves drift in the prograde direction.

First of all our results were compared with those of Zhang \& Liao (2004), and Zhang et al. (2007). The transformations for the critical Rayleigh number $R a_{c}^{Z}=R a_{c} / \sqrt{T a}$ and the critical precession frequency $\omega_{c}^{Z}=\omega_{c} / \sqrt{T a}$ were used, to take into account the different non-dimensional scales of the authors. Some of the values checked are given in table 1 . The critical parameters agree very well when $T a=10^{8}$, and only slight discrepancies are found for $\sigma=0.023$. In this case the critical wave number is different, but the Rayleigh number for $m=8$ is $3.203 \times 10^{6}$, very close to that of our $m_{c}=7$ mode, with $\omega_{c}=-2.489 \times 10^{4}$, which agrees very well with that of Zhang \& Liao (2004). Moreover, both eigenfunctions are equatorially attached, so different codes and numerical truncation errors could justify the small differences.

\section{Taylor number dependence}

Figure 1 shows the Taylor number dependence of $R a_{c}$, minus $\omega_{c}$, and $m_{c}$ for $\sigma=0.01$, representative of very low Prandtl numbers. In the first figure, the left axis and the magenta curve correspond to the Rayleigh parameter, while the blue curve and the right axis indicate the drifting frequency. The marginal curves are the envelope of more than 50 curves, one for each of the wave numbers taken and shown in the second figure.

There are two regimes. For Taylor numbers below $6.31 \times 10^{10}, R a_{c}$ increases linearly with the Taylor number, and $\omega_{c}$ goes as the square root. At higher $T a, R a_{c}$ and $\omega_{c}$ follow potential laws different from those predicted for the asymptotic theory of large $\sigma$. Initially, the preferred wave number is $m_{c}=1$. The preference of this mode of convection is in agreement with that found by Busse \& Simitev (2004). Afterwards, it jumps to $m_{c}=8$ and increases monotonically following a staircase. For the Prandtl number used, the selected laws are $R a_{c}=9635+5.879 \times 10^{-5} \mathrm{Ta}, \omega_{c}=-0.175 T a^{0.500}$ if $m_{c}=1$, and $R a_{c}=2.85 \times 10^{-3} \mathrm{Ta}^{0.845}, \omega_{c}=-1.58 T a^{0.428}$ and $m_{c}=1.71 \times 10^{-2} \mathrm{Ta}^{0.251}$ (magenta solid line) for the highest $T a$.

The first row of figure 2 shows the contour plots of the temperature perturbation, the second of the azimuthal velocity, $v_{\varphi}$, and the third of the kinetic energy density, $E_{K}=\|\boldsymbol{v}\|^{2} / 2$. Each set of three figures includes projections of the fields on a spherical surface, and on the equatorial and a meridional plane. The lines marked in each surface indicate the position where the other two projections are taken. The left three columns correspond to the critical $m_{c}=1$ mode of convection. The right columns show the critical inertial $m_{c}=50$ equatorially-attached mode of convection, of the type described in Zhang (1993). The size of the equatorial waveguide agrees very well with that of the inertial solutions found in that paper: consequently, due to the high wave number the conduction state is stable in almost all of the body of the fluid.

The contour plots of both solutions have a symmetric $\Theta$ with respect to the equator, and $v_{\varphi}$ and $E_{K}$ have their maxima in the equatorial plane at $r=r_{o}$. The temperature perturbation of the $m=1$ pattern, fills the domain, while that of the EA modes is strongly trapped in the outer surface. In both cases most of the contribution to the kinetic energy density comes from the azimuthal velocity.

\section{Prandtl number dependence}

Figure 3 shows the dependence of the critical parameters with the Prandtl number for a large Taylor number. In this case four different behaviours are distinguished in the range computed. Below approximately $\sigma=6 \times 10^{-6}$, the preferred modes are axisymmetric 

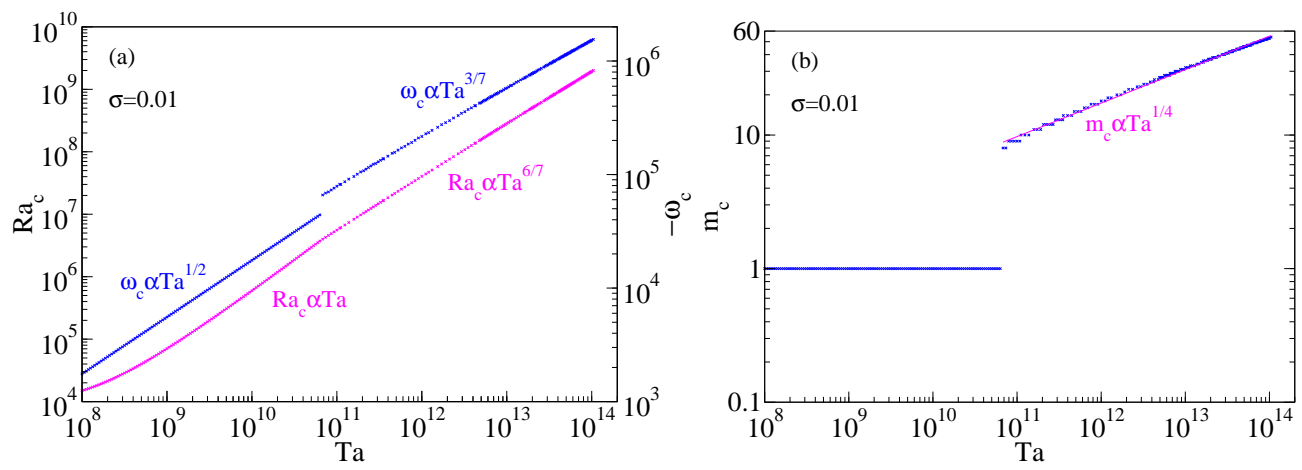

Figure 1. Critical a) Rayleigh number (magenta) and minus the precession frequency (blue), and b) azimuthal wave number versus the Taylor number.
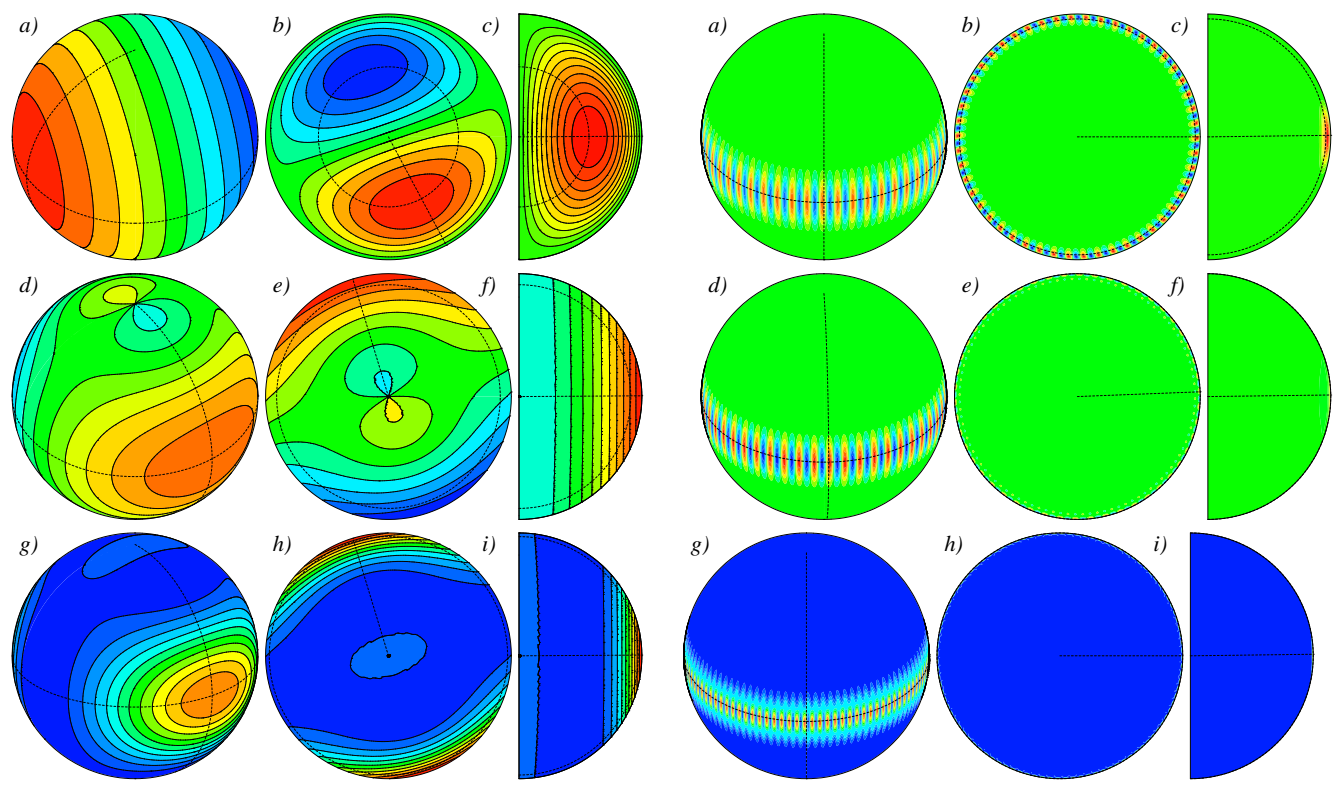

Figure 2. (a-c) Contour plots of $\Theta$, (d-f) $v_{\varphi}$, and (g-i) $E_{K}$, for two solutions. The left three columns for the inertial $m_{c}=1$ mode with $\sigma=0.01, T a=4.47 \times 10^{10}, R a_{c}=2.60 \times 10^{6}$ and $\omega_{c}=-3.71 \times 10^{4}$. Those at right are for an EA mode of $m_{c}=50$ and $\sigma=0.01, T a=7.56 \times 10^{13}$, $R a_{c}=1.53 \times 10^{9}$ and $\omega_{c}=-1.36 \times 10^{6}$. Green means zero for $\Theta$ and $v_{\varphi}$, and blue means zero for $E_{K}$. The dashed lines marked on each surface indicate the position where the other two projections are taken. Those spherical and meridional almost cut the maximum value of the cells and vortices.

with oscillatory frequencies almost an order of magnitude higher than that of the other inertial modes. Notice that this mode of convection cannot be described as a perturbed solution of an inertial wave since it does not travel. Next, up to approximately $1.10 \times 10^{-3}$, the preferred eigenmode has constant azimuthal wave number $m_{c}=1$ with the pattern of convection shown before. From approximately $1.10 \times 10^{-3}$ to $1.06 \times 10^{-2}$, the preferred modes are EA. In this transition the critical wave number jumps from 1 to 8 and then increases up to 27 . Finally, near $1.06 \times 10^{-2}$ the wave number falls from 27 to 14 in a third transition. By increasing $\sigma$, there is, initially, a small transition zone where the eigenfunctions retain characteristics of the EA modes in the sense that the kinetic energy density remains maximal at the equator, but the cells of convection spread to the interior 


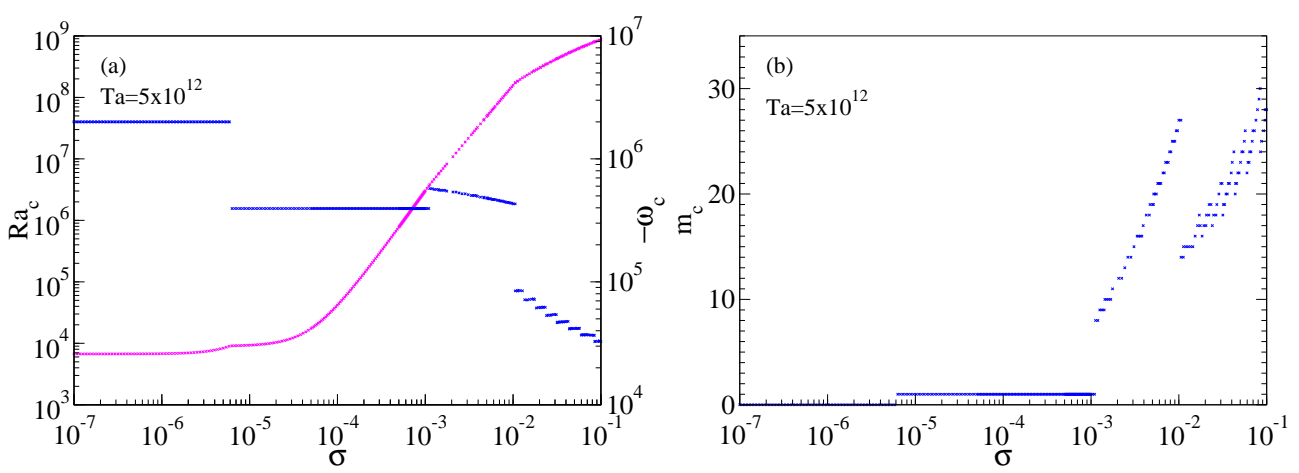

Figure 3. Critical a) Rayleigh number (magenta) and minus the precession frequency (blue), and b) azimuthal wave number versus the Prandtl number.
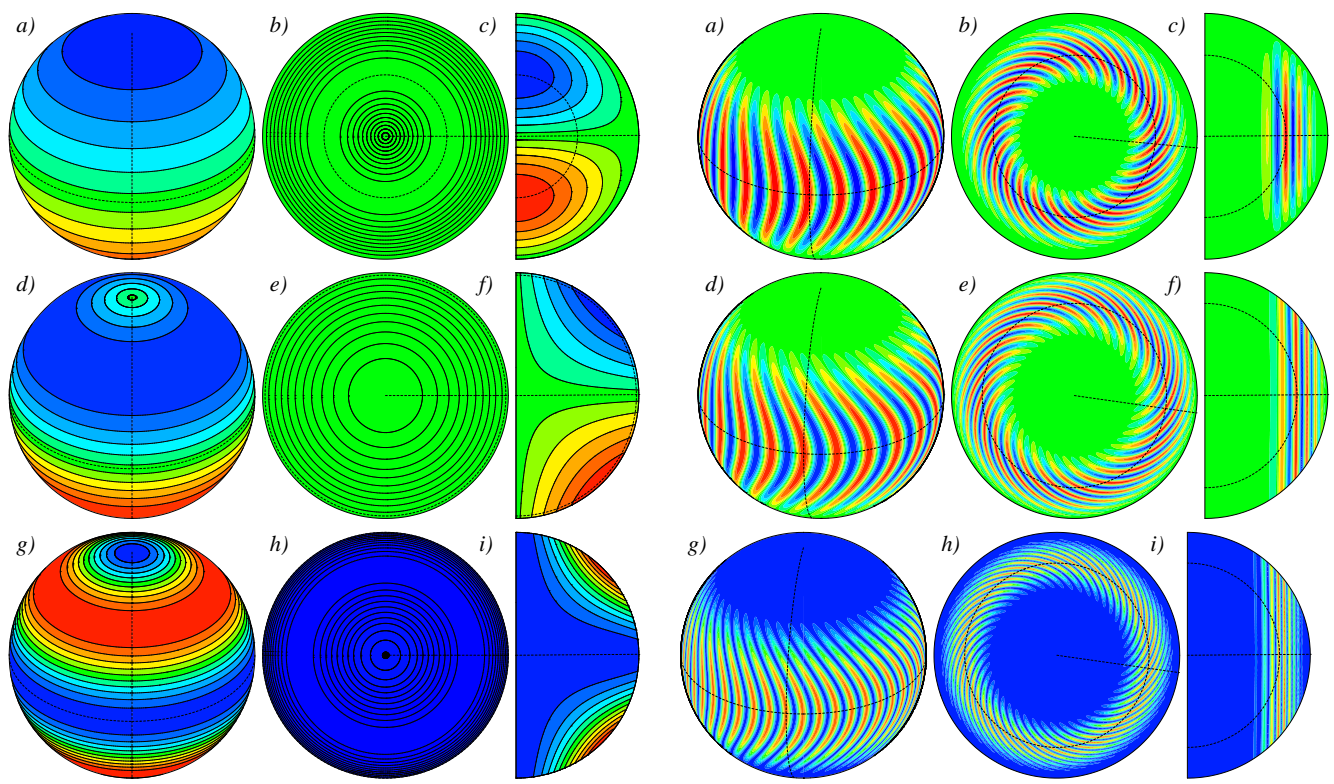

Figure 4. (a-c) Contour plots of $\Theta$, (d-f) $v_{\varphi}$ and (g-i) $E_{K}$, for two solutions. The left three columns for the axisymmetric mode, with $T a=5 \times 10^{12}, \sigma=10^{-7}, R a_{c}=6.72 \times 10^{3}$, $\omega_{c}=-2.00 \times 10^{6}$. Those at right are for a SP mode of $m_{c}=23$ and $T a=5 \times 10^{12}, \sigma=5.03 \times 10^{-2}$, $R a_{c}=5.84 \times 10^{8}, \omega_{c}=-4.18 \times 10^{4}$. The projections are selected following the same criteria as in figure 2 .

of the sphere, detaching progressively from the outer surface and spiralling, but forming several humps in the interior of the spirals. These linear solutions were first described in spherical shells by Ardes et al. (1997). From $\sigma \approx 0.02$ the preferred modes are already spiralling columnar and detached from the equator. Then the wave number increases, making a zigzag, so from $m_{c} \gtrsim 17$ there are different preferred spiralling patterns of convection with the same azimuthal wave number, which differ in the radial location, width and curvature of the spirals.

In this case the type of law fulfilled by the critical parameters in each interval is not always potential. When $m_{c}=1$, while the precession frequency is constant $\left(3.95 \times 10^{5}\right)$, the Rayleigh number follows the quadratic law $R a_{c}=7.699 \times 10^{3}+4.759 \times 10^{7} \sigma+$ $2.976 \times 10^{12} \sigma^{2}$ only from $\sigma=10^{-4}$. For the equatorially-attached modes, power laws 

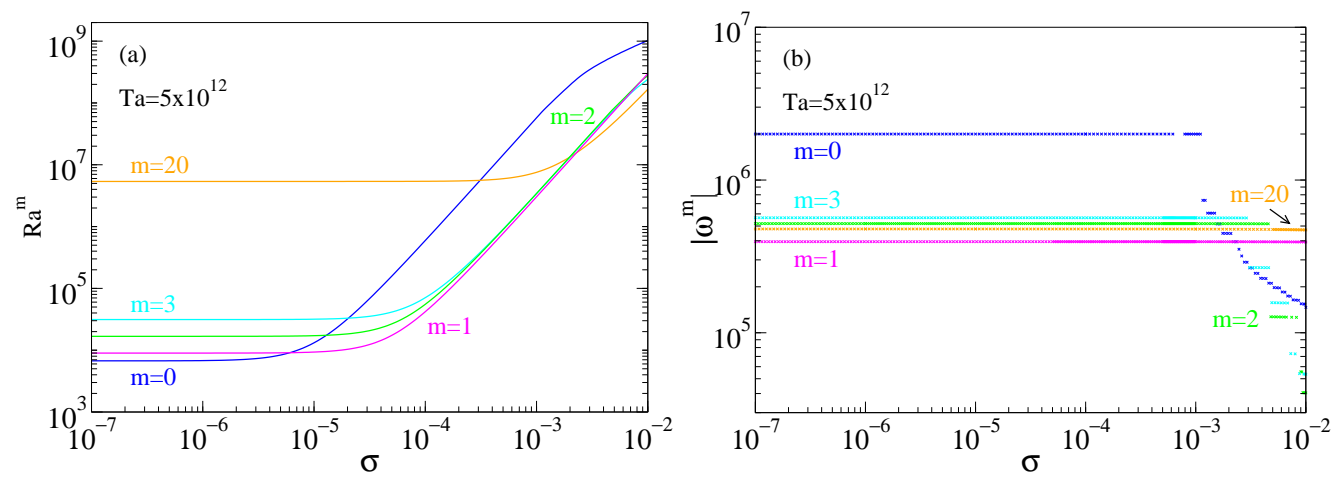

Figure 5. a) Critical Rayleigh number and b) critical absolute value of the frequency of the modes $m=0,1,2,3$ and 20 versus the Prandtl number.

$R a_{c}=3.98 \times 10^{11} \sigma^{1.70}, \omega_{c}=-2.40 \times 10^{5} \sigma^{0.13}$ and $m_{c}=332.4 \sigma^{0.54}$ are obtained . Finally, in the fourth interval, the critical Rayleigh number goes as $R a_{c}=5.553 \times 10^{7}+$ $1.315 \times 10^{10} \sigma-5.140 \times 10^{10} \sigma^{2}$ and the precession frequency as $\omega_{c}=-1.143 \times 10^{4} \sigma^{0.44}$.

In figure 4 contour plots of the axisymmetric mode and one of the $m_{c}=23 \mathrm{SP}$ modes found are represented. They correspond to the first and last intervals of figure 3 . The former has $\Theta$ and the components $v_{r}$ and $v_{\varphi}$ of the velocity field antisymmetric with respect to the equator and $v_{\theta}$ is symmetric. This mode is described with more detail in the next section. For the latter, the symmetries of these fields are interchanged and $E_{K}$ is columnar.

\section{The Low-Prandtl-number limit}

To illustrate what happens in the low-Prandtl-number limit and how the axisymmetric mode becomes preferred, the four lowest neutral curves at $\sigma=10^{-7}$, i.e. those of $m=$ $0,1,2,3$, plus that of $m=20$, just as an example, are represented in figure 5 . The first figure shows the Rayleigh number versus $\sigma$, and the second the absolute value of the frequency. Notice that the envelope of this curves does not give $R a_{c}$ since, as it was seen before, at $T a=5 \times 10^{12}$, the $m_{c}=1$ mode is replaced by an EA mode with $m_{c}=8$.

When a mode reaches the $\sigma$-zero limit, $R a^{m}$ and $\omega^{m}$ become constant. At $\sigma=10^{-2}$ the Rayleigh number of the axisymmetric mode is more than an order of magnitude higher than the critical value, but since it reaches the asymptotic limit (independence of $R a^{m}$ with $\sigma$ ) at a lower value of $\sigma$ than the other $m$ and the slopes of $R a^{m}$ versus $\sigma$ are similar before the limit, it becomes preferred.

Figure 6 shows a sequence of projections of the contour plots of $\Theta$ and all of the components of the velocity field, $\left(v_{r}, v_{\theta}, v_{\varphi}\right)$, onto a meridional plane in a period of oscillation, for $\sigma=10^{-7}$. The axisymmetric velocity field consists in the superposition of a single vortex in the $(r, \theta)$ plane, which fills the meridional section, and a transverse azimuthal circulation, antisymmetric with respect to the equator. Both components oscillate periodically in time with a phase shift of $\pi / 2$, i.e.

$$
\begin{aligned}
v_{r}(t, r, \theta) & =v_{r}^{0}(r, \theta) \cos (\omega t+\delta), \\
v_{\theta}(t, r, \theta) & =v_{\theta}^{0}(r, \theta) \cos (\omega t+\delta), \\
v_{\varphi}(t, r, \theta) & =v_{\varphi}^{0}(r, \theta) \sin (\omega t+\delta), \\
\Theta(t, r, \theta) & =\Theta^{0}(r, \theta) \cos (\omega t+\delta) .
\end{aligned}
$$




\begin{tabular}{lllllllll}
\hline $\begin{array}{l}\text { Fraction } \\
\text { of period }\end{array}$ & $v_{r}$ & $v_{\theta}$ & $v_{\varphi}$ & $\Theta$ & $\begin{array}{c}\text { Fraction } \\
\text { of period }\end{array}$ & $v_{r}$ & $v_{\theta}$ & $v_{\varphi}$ \\
\hline
\end{tabular}

0

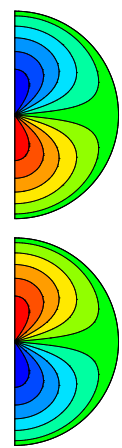

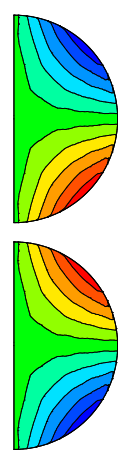

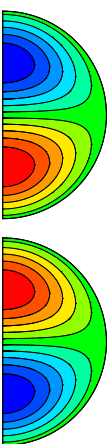

$1 / 4$

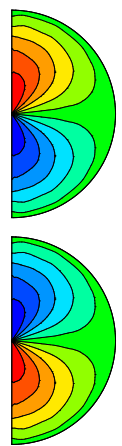

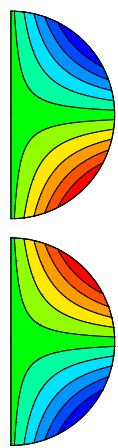

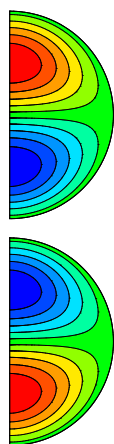

FiguRE 6. Snapshots of the meridional projection of the contour plots of the preferred axisymmetric mode for $\sigma=10^{-7}, T a=5 \times 10^{12}, R a_{c}=6.72 \times 10^{3}$, and $\omega_{c}=-2.00 \times 10^{6}$.

The oscillation of the vortex consists in its change in amplitude and sense of rotation, maintaining its shape, and that of $v_{\varphi}$ in the alternation of the prograde and retrograde velocities between the two hemispheres. Then, its mean zonal flow integrated over an oscillation period is zero. This type of axisymmetric mode is known as torsional. Since $v_{r}, v_{\theta}$ and $v_{\varphi}$ are out of phase and the maxima are of the same order, the maximum kinetic energy density is localised in the interior of the sphere and in a thin band of the surface at low latitudes when $v_{r}, v_{\theta}$ have maxima, and in the surface at mid-latitudes when $v_{\varphi}$ is the dominant component.

Torsional oscillations in rapidly rotating systems were studied by Liao \& Zhang (2006) and Liao et al. (2007) in a rotating annular channel uniformly heated from below. The authors developed an asymptotic theory (confirmed by numerical calculations) describing the mechanism for the excitation and maintenance of the oscillations. According to them, the Coriolis force provides the restoring force, and the heating the energy necessary to sustain the oscillations against viscous dissipation. Moreover, they demonstrate, in the range of parameters explored in the present paper $(T a \gg 1$ and $\sigma \ll 1)$, that nonlinear torsional oscillations can be maintained by thermal convection at supercritical $R a$.

In our case the instability giving rise to torsional modes is purely hydrodynamic as in Liao et al. (2007). The existence of azimuthal velocity in the linear regime can be easily understood by looking at equations (2.1-2.3) for the axisymmetric modes in the low-Prandtl number limit $\left(\partial_{\varphi}=0, \sigma \rightarrow 0\right)$, and at the expression of the velocity field in terms of the potentials, $v_{r}=-\left(\sin \theta \partial_{\theta \theta}^{2} \Phi+\cos \theta \partial_{\theta} \Phi\right) / r \sin \theta, v_{\theta}=\partial_{r \theta}^{2}(r \Phi) / r$, and $v_{\varphi}=-\partial_{\theta} \Psi$. Since $v_{\varphi}$ depends only on $\Psi$, as soon as a non-zero meridional velocity field appears $(\Phi \neq 0)$ due to the heating, the Coriolis force generates an azimuthal velocity of different sign in each hemisphere. Moreover, at critical values of the parameters the time dependence is $X(t, r, \theta)=\exp (i \omega t) X(r, \theta)$, so by replacing $\partial_{t}$ by $i \omega$ in the equations it is clear that there is a phase lag of $\pi / 2$ between $\Phi$ and $\Psi$, or, which is the same, between $v_{r}, v_{\theta}$ and $v_{\varphi}$.

By considering the annulus as a polar slice of a spherical shell (where gravity is almost parallel to the rotation vector), it is possible to relate the critical parameters of both geometries. By defining $d$ as the depth of the annulus and $g$ as its constant gravity, it results that $T a_{L}^{1 / 2}=\left(d / r_{o}\right)^{2} T a^{1 / 2}$. The subscript $L$ means that parameters are scaled 
as in Liao \& Zhang (2006) and Liao et al. (2007). They deduce that for $\sigma T a_{L}^{1 / 2}=0.1$ the most unstable mode is axisymmetric and oscillatory when $T a \gg 1$. By estimating $d=r_{o} / 10$, the above relations give $\sigma T a^{1 / 2}=10$. Our calculations show the beginning of axisymmetric oscillations at $\sigma T a^{1 / 2} \approx 13$ (see figure 3). Taking into account the inaccurate estimation, the result agrees very well with the relation found in the annulus. Moreover, the order of magnitude of the frequency of the oscillations is the same: for instance, with $\omega_{L}=\omega / T a^{1 / 2}$ and the values of figure 5, i.e. for $T a=5 \times 10^{12}$ and $\omega=2 \times 10^{6}, \omega_{L}=0.89$, which is close to $\omega_{L}=1.4$, given in Liao \& Zhang (2006) for $T a=10^{10}$. However, as it is discussed in the conclusions of the paper, we have not been able to find preferred torsional modes for $\sigma=0.01$ at high Taylor numbers.

\section{Conclusions}

The linear stability analysis of the onset of convection for high $T a$ and low $\sigma$ has shown that there are two different transitions between inertial modes, and a third one between the inertial EA and the convective SP modes.

From the linear analysis for $T a \gg 1$, power-law dependences $R a_{c} \sim T a^{6 / 7}, \omega_{c} \sim T a^{3 / 7}$ and $m_{c} \sim T a^{1 / 4}$, and $R a_{c} \sim \sigma^{1.70}, \omega_{c} \sim \sigma^{0.13}$ and $m_{c} \sim \sigma^{0.54}$ for the critical parameters of the EA modes have been determined.

The numerical results of figures 1 and 3 seem to indicate that when $T a=5 \times 10^{12}$ $(T a \gg 1)$, torsional patterns of convection are preferred when $\sigma T a^{1 / 2} \lesssim 13.4$, i.e. for values of $T a$ two orders of magnitude higher than the reciprocal of $\sigma$. This mode has an oscillatory frequency that is almost an order of magnitude higher than the precession frequency of the $m=1$ mode, preferred in the range $13.4 \lesssim \sigma T a^{1 / 2} \lesssim 2.24 \times 10^{3}$, and than that of the EA modes, critical in $2.24 \times 10^{3} \lesssim \sigma T a^{1 / 2} \lesssim 2.37 \times 10^{4}$.

In order to elucidate the range of parameters where torsional modes could be preferred, and how the zero-limit evolves at higher Taylor numbers we have computed some curves of the $m=0,1,2$ modes. Specifically, liquid metals $(\sigma=0.01)$ at lower $T a$ than those shown in figure $3, \sigma=10^{-7}$ (first point of figure 5) and $T a>5 \times 10^{12}$, and $\sigma=5 \times 10^{-4}$ and $T a<5 \times 10^{12}$ have been used. In the first case the $m=0$ mode is only found to be preferred for $T a<1.56 \times 10^{6}$, with oscillating frequencies that are also one order of magnitude higher than those of the $m=1$ mode. The pattern of convection is that shown in figure 4 . In the second case no mode crossing has been found. From $T a=5 \times 10^{12}$ to $T a=10^{15}$ the critical $R a$ of the $m=0$ mode increases by less than $2 \%$ while that of the $m=1$ mode decreases by less than $7 \%$. This means that following this tendency the zero$\sigma$ limit would persist in the range of parameters of geophysical and astrophysical interest. The corresponding frequencies change from $2.00 \times 10^{6}$ to $2.83 \times 10^{7}$ at $T a=10^{15}$. In the last case the modes $m=0,1$ cross at $T a=7.20 \times 10^{8}$. The results seem to confirm that the antisymmetric torsional mode crosses the symmetric $m=1$ mode for $\sigma T a^{1 / 2}=O(10)$.

Since the axisymmetric mode can be excited at very low Rayleigh numbers with very high frequencies, its contribution to the nonlinear dynamics could be relevant to understanding the generation and maintenance of non-axisymmetric planetary magnetic fields. Moreover, as was argued by Landeau \& Aubert (2011), even if the inertial and the axisymmetric modes are not the first to become unstable, they can influence the nonlinear regimes.

The comparison of the results with those obtained from codes for rotating spherical shells (see, for instance, Net et al. 2008) shows that the critical parameters for the SP and EA solutions with high wave numbers can be well approximated from large finitegap numerical computations $\left(r_{i} / r_{o}<0.01\right)$. However, solutions with low wave numbers (which are preferred at very low $\sigma$ ) require the problem to be solved in the full sphere. 
We hope that the numerical results of this paper will help in developing a definitive theory of the onset of convection for the very small Prandtl numbers.

This research has been supported by the Spanish MCYT/FEDER project FIS201340674, and Catalan GENCAT project 2014-SGR-1145.

\section{REFERENCES}

Al-Shamali, F.M., Heimpel, M.H. \& Aurnou, J.M. 2004 Varying the spherical shell geometry in rotating thermal convection. Geophys. Astrophys. Fluid Dynamics 98 (2), 153-169.

Ardes, M., Busse, F. H. \& Wicht, J. 1997 Thermal convection in rotating spherical shells. Phys. Earth Planet. Inter. 99, 55-67.

Bassom, A. P., Soward, A. M. \& Starchenko, S. V. 2011 The onset of strongly localized thermal convection in rotating spherical shells. J. Fluid Mech. 689, 376-416.

Busse, F. H. 1970 Thermal instabilities in rapidly rotating systems. J. Fluid Mech. 44, 441-460.

Busse, F. H. 2002 Convective flows in rapidly rotating spheres and their dynamo action. Phys. Fluids 14 (4), 1301-1313.

Busse, F. H. \& Simitev, R. 2004 Inertial convection in rotating fluid spheres. J. Fluid Mech. 498, 23-30.

Carrigan, C. R. \& Busse, F. H. 1983 An experimental and theoretical investigation of the onset of convection in rotating spherical shells. J. Fluid Mech. 126, 287-305.

Dormy, E., Soward, A. M., Jones, C. A., Jault, D. \& CARdin, P. 2004 The onset of thermal convection in rotating spherical shells. J. Fluid Mech. 501, 43-70.

Garcia, F., SÁnchez, J. \& Net, M. 2008 Antisymmetric polar modes of thermal convection in rotating spherical fluid shells at high Taylor numbers. Phys. Rev. Lett. 101 (19), 194501.

Jones, C. A., Soward, A. M. \& Mussa, A. I. 2000 The onset of thermal convection in a rapidly rotating sphere. J. Fluid Mech. 405, 157-179.

LANDEAU, M. \& AuberT, J. 2011 Equatorially asymmetric convection inducing a hemispherical magnetic field in rotating spheres and implications for the past martian dynamo. Phys. Earth Planet. Inter. 185, 61-73.

Lehoucq, R. B. \& Sorensen, D. C. 1996 Deflation techniques for an implicitly restarted Arnoldi iteration. SIAM J. Matrix Anal. Appl. 17, 789-821.

LiaO, X. \& Zhang, K. 2006 On the convective excitation of torsional oscillations in rotating systems. Astrophys. J. 638 (2), L113-L116.

LiaO, X., Zhang, K. \& ChAng, Y. 2007 Nonlinear torsional oscillations in rotating systems. Phys. Rev. Lett. 98, 094501.

Net, M., Garcia, F. \& SÁnchez, J. 2008 On the onset of low-Prandtl-number convection in rotating spherical shells: non-slip boundary conditions. J. Fluid Mech. 601, 317-337.

Roberts, P. H. 1968 On the thermal instability of a rotating fluid sphere containing heat sources. Phil. Trans. R. Soc. Lond. A 263 (1136), 93-117.

SÁnchez, J., Garcia, F. \& Net, M. 2016 Radial collocation methods for the onset of convection in rotating spheres. J. Comput. Phys. 308, 273-288.

YANO, J. I. 1992 Asymptotic theory of thermal convection in a rapidly rotating system. J. Fluid Mech. 243, 103-131.

ZHANG, K. 1992 Spiralling columnar convection in rapidly rotating spherical fluid shells. J. Fluid Mech. 236, 535-556.

ZhANG, K. 1993 On equatorially trapped boundary inertial waves. J. Fluid Mech. 248, 203-217.

Zhang, K. 1994 On coupling between the Poincaré equation and the heat equation. J. Fluid Mech. 268, 211-229.

Zhang, K., Earnshaw, P., Liao, X. \& Busse, F.H. 2001 On inertial waves in a rotating fluid sphere. JFM 437, 103-119.

ZhANG, K. \& LiAO, X. 2004 A new asymptotic method for the analysis of convection in a rapidly rotating sphere. J. Fluid Mech. 518, 319-346.

Zhang, K., LiaO, X. \& Busse, F.H. 2007 Asymptotic solutions of convection in rapidly rotating non-slip spheres. J. Fluid Mech. 578, 371-380. 